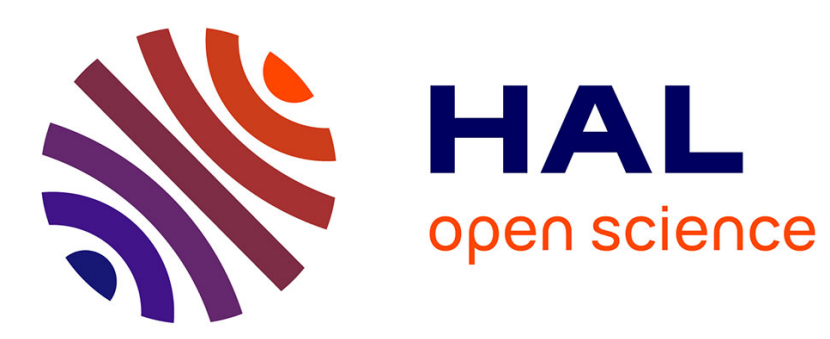

\title{
Bayesian network for the prediction of situation awareness errors
}

Jean Marc Salotti

\section{To cite this version:}

Jean Marc Salotti. Bayesian network for the prediction of situation awareness errors. International Journal of Human Factors Modelling and Simulation, 2018, 6 (2/3), pp.119-126. 10.1504/IJHFMS.2018.093174 . hal-01944420

\section{HAL Id: hal-01944420 \\ https://hal.inria.fr/hal-01944420}

Submitted on 5 Dec 2018

HAL is a multi-disciplinary open access archive for the deposit and dissemination of scientific research documents, whether they are published or not. The documents may come from teaching and research institutions in France or abroad, or from public or private research centers.
L'archive ouverte pluridisciplinaire HAL, est destinée au dépôt et à la diffusion de documents scientifiques de niveau recherche, publiés ou non, émanant des établissements d'enseignement et de recherche français ou étrangers, des laboratoires publics ou privés. 


\title{
Bayesian Network for the Prediction of Situation Awareness Errors
}

\author{
Jean-Marc Salotti
} Laboratoire de 1'Intégration du Matériau au Système, UMR CNRS 5218, équipe Auctus INRIA,
ENSC, Bordeaux University, IPB, jean-marc.salotti@ensc.fr

\begin{abstract}
A new method is proposed to predict situation awareness errors in training simulations. It is based on Endsley's model and the 8 "situation awareness demons" that she described. The predictions are determined thanks to a Bayesian network and Noisy-Or nodes. A maturity model is introduced to come up with the initialization problem. The NASA behavioural competency model is also used to take individual differences into account.
\end{abstract}

Keywords: Situation awareness, Bayesian network, noisy-or, behavioural competency

\section{Introduction}

Situational awareness (SA) has been studied by many authors (Muniz et al 1998; De Winter et al 2014; Endsley 2010; 2016; Sarter and Woods 1991). A possible definition is to be aware of what is happening around and what information means in the current context and for the future. If for any reason there is a SA error, the performance of the current task might decline, a wrong action might be taken and the life of several persons might be put in danger. SA is of particular importance in aeronautics, astronautics, nuclear industry or military operations, where operators have to consider a lot of information with time constraints and complex decision making. While lots of effort has been put on failure analysis, objective and subjective measurements during training or real time operations, it is still difficult to characterize the risk of SA degradation and to predict human errors. As SA degradation is often linked with workload and human capabilities, a possible approach is to estimate the mental workload and the human capacity factor in a double exponential probability distribution function (Suhir 2013, 2015). However, the workload does not include all cases of situation awareness problems. For instance, an inappropriate action might be taken because the operator was following a wrong mental model during a low workload session. The framework of Bayesian networks is often used to assess SA degradation and predict operational risks (Mkrtchyan et al 2015). It is proposed here to examine how they can be used during training in order to predict the evolution of human performance. In section 2, Endsley's model is described and discussed. In Section 3, Bayesian networks are revisited and a new approach is presented.

\section{Situational awareness assessment}

\subsection{Situational awareness}

Endsley proposed a model to explain situational awareness issues (Endsley 2000; 2016). 3 levels are considered:

- First level: perception of the elements of the environment, situation status, attributes of relevant variables, dynamics and context.

- Second level: comprehension of the current situation. Data and cues have to be integrated and understood in order to build a clear and relevant representation of the situation.

- Third level: projection of future status. It is important to predict the dynamics of the systems and the impact of possible actions to solve a problem or complete a task.

According to Endsley, different mechanisms or causes are possibly at work to alter one of several SA levels (Endsley 2012). They are called "SA demons". These mechanisms are classified in 8 categories. Some categories are linked to data, which can exceed human processing capabilities, be too complex or 
with inadequate saliency. Other categories concern inappropriate human behaviors, due to stress, inattention, misleading or immature mental models, misconception of interface or inappropriate work sharing with automated systems or team members. The 8 main causes are displayed in the graph that is presented figure 1 .

According to other authors, Endsley's theory of situation awareness does not take orienting activity into account (Bedny 2014). Orienting activity includes cognitive mechanisms such as goal definition, motivation, emotional dependences, creativity, etc. It is a complex self-regulative process that is often tightly linked to the task. Orienting activity does not impact SA directly but it participates to the activation of specific demons. To some extent, the impact is taken into account in the workload, the fatigue, the stress, the attentional capacity and the mental models.

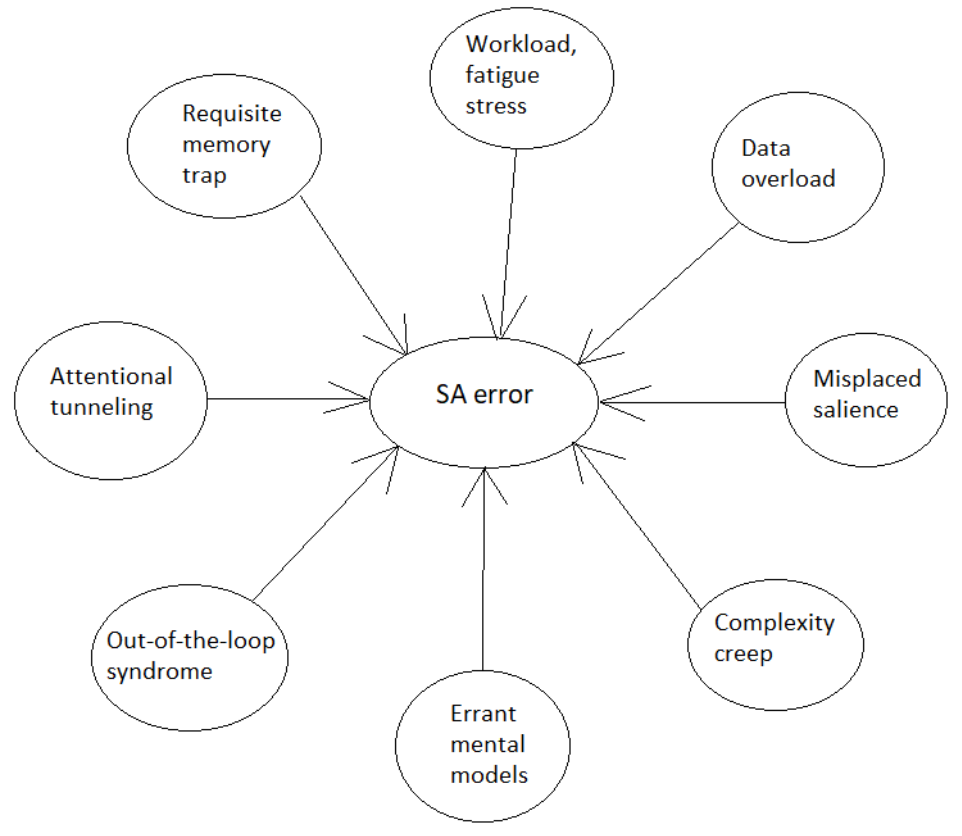

Figure 1: Possible causes of SA errors according to Endsley.

\subsection{Training}

As it is outlined by Endsley, an important issue is to develop correct and accurate mental models of the processes, relationships and dynamics of the variables (Endsley 2016). Training improves human's ability to comprehend a dangerous and complex situation, the impact of each variable, time constraints and the correct sequence of actions that have to be carried out. Training cannot help in reducing the workload or the complexity of a situation and if the interface of the systems provides incomplete or misleading information, even the most talented human could fail. However, training may help in reducing the risks associated to attentional tunneling, misplaced salience, fatigue, errant mental models or out-of-the-loop syndrome. To this end, it is important to define simulations for situation awareness oriented training (Endsley 2016; Muniz et al 1998). Most mental models that need to be acquired relate to the application. However, training also allows improvements of generic behaviors when facing the problems. For the training of astronauts going to the International Space Station, NASA is using a Human Behavior and Performance Competency Model (NASA 2008). During Extra-Vehicular Activity (EVA) simulations, human factors experts assess their performance according to numerous and accurate competencies for communicating, monitoring their stress and fatigue, solving problems, maintaining 
attention to relevant parameters, team working, etc. In this approach, the loss of situation awareness can be mitigated by the acquisition of these generic behavioral competencies.

An important issue during the training phase is the assessment of the trainees. An accurate assessment of each simulation is possible. However, after each of them, there is in general a discussion with the trainees to help them understand the errors, the relevant parameters, the actions that were expected, etc. Trainees also have their personal feedback of the experiment, which allows them to improve their performance for the next trial. The assessment therefore corresponds to the level of the trainee during the simulation, but it is already out of date and does not correspond to the current level. This problem will be further discussed in the next sections.

\subsection{Bayesian networks}

The Bayesian network framework provides mathematical tools to calculate conditional probabilities in order to determine the risk that a SA error occurs $(\overline{S A})$. Formally, a Bayesian network is an acyclic oriented graph, which can be defined by $\mathrm{G}=(\mathrm{N}, \mathrm{A})$, where $\mathrm{N}$ is the set of nodes and A the set of arcs representing conditional dependencies (Pearl 1988). For each node $\mathrm{x}$ of $\mathrm{N}$, a joint conditional probability distribution is associated: $\mathrm{P}(\mathrm{x} \mid \mathrm{pa}(\mathrm{x}))$, where $\mathrm{pa}(\mathrm{x})$ represents the knowledge on the parent nodes.

Two important problems have to be addressed to build a Bayesian network. The first one is to determine the structure of the network and the second is to determine the joint probabilities associated to each node. In general, the structure of the network is defined by a group of experts who have a good knowledge of the causal links among the variables and the events. Another approach is possible if a large database of events and data is available. The parent nodes of a given node can be determined according to a careful analysis of data and events that occurred just before the event characterizing a change of status for that node. In the context of our study, a node of the network might represent a human error due to loss of situation awareness and the parent nodes might be actions or events that occurred just before. The second problem is to determine the joint probabilities associated to the network. They can be determined empirically by a team of experts or calculated using a database of past experiments. Numerous methods and applications of Bayesian networks have been proposed for human reliability analysis or human error prediction (Baoping et al 2013; Barua et al 2016; Groth and Mosley 2011; Park et al 2013). A review of Bayesian belief networks has been carried out by Mkrtchyan et al (2015).

A major drawback of Bayesian networks is the complexity of the graph and the numerous calculations that have to be performed to determine joint probabilities. It is especially an issue if the number of parent nodes is high, as it becomes impractical to identify and calculate all values. In order to simplify the problem, some assumptions are often made. For instance, if it is possible to assume that all causes are independent from each other and if all of them have been identified, the Noisy-Or model (Pearl 1988; Lemmer 2005; Salotti 2010) can be used (equation (1)). In this case, only n probabilities have to be calculated for $\mathrm{n}$ parent nodes.

$$
P\left(\overline{S A} / X_{1} \ldots X_{\mathrm{n}}\right)=1-\prod_{i=1}^{\mathrm{n}}\left(1-P\left(X_{i}\right)\right)
$$

\section{A new method to predict situation awareness errors during training}

\subsection{Implementation of a Bayesian network}

It is proposed here to define the 8 possible "SA demons" described by Endsley as 8 parameters with a causal link to "situation awareness error" and to use them in a Bayesian network. The graph in Figure 1 
therefore depicts that network. An important issue is to estimate each parameter. A simple approach is to ask a team of experts to supervise the activities and to provide an assessment with a small number of discrete values, such as "not observed, small, strong". It is possible to define objective measurements provided by the application (specific state or event, unexpected action, etc.), to proceed to interviews and to exploit trainees' responses. A complementary approach is to use physiological data, especially for stress estimation.

Another issue concerns the number of joint probabilities and their calculation. As that number would be high, a tremendous number of training sessions would be required to obtain sufficient data and compute the conditional probabilities. The Noisy-Or model is an interesting tradeoff. This simplification might be unjustifiable if the combination of several causes drastically increase the risk of human errors. Indeed, it is well-known that humans can usually come up with situations where only one problem occurs at a time, while they would systematically fail if another problem comes into play. This is due to the mobilization of cognitive and attentional resources for problem solving and the difficulty to switch the different contexts. However, this case can be taken into account. As there is a node dedicated to "attentional tunneling", it can be assumed that this node includes all situation awareness demons if attentional tunneling is part of them. In practice, it means that "attentional tunneling" must be triggered alone. In every other situation, a standard Noisy-Or approach can be used.

\subsection{Initialization problem}

If a large database of past simulations is available for each scenario, the probability associated to the observation of each cause can easily be calculated. However, this database is not available during the first simulations and the performance also depends on the experience of the participants. It is in general expected that participants improve their scores for each new simulation. These two important problems can be solved using a maturity model, as it is often used in astronautics (Salotti and Suhir 2014), see equation (2).

$$
P(x)=P_{\max }(x)+\left(P_{i}(x)-P_{\max }(x)\right) \times\left(\left(n_{0}-n\right) / n_{0}\right)^{k}
$$

where: $\mathrm{P}(\mathrm{x})$ is the probability of not observing $\mathrm{x}$ (one of the 8 causes) after $\mathrm{n}$ simulations

$\mathrm{P}_{\max }(\mathrm{x})$ is the final probability of not observing $\mathrm{x}$ for a trained expert

$\mathrm{P}_{\mathrm{i}}(\mathrm{x})$ is the initial probability of not observing $\mathrm{x}$ (if $\mathrm{n}=0 \mathrm{P}(\mathrm{x})=\mathrm{P}_{\mathrm{i}}(\mathrm{x})$ )

$\mathrm{n}_{0}$ is the number of simulations to become an expert

$\mathrm{k}$ is a decay constant

For the first simulation, $\mathrm{P}_{\mathrm{i}}(\mathrm{x})$ can be set empirically by an expert of the domain. In general, $\mathrm{P}_{\max }(\mathrm{x})$ can be set to 1 , but if important difficulties are integrated in the scenario, situation awareness problems may be observed even during simulations with experts. In this case, $\mathrm{P}_{\max }(\mathrm{x})$ may be set to lower values. $\mathrm{n}_{0}$ depends on the complexity and variability of the simulations. It also has to be set by experts of the domain. If the scenario is constantly repeated with the same parameters and if the debriefing is efficient, it is in general very small (e.g., 2 or 3 ). k corresponds to the learning speed. With high values (e.g., 3 or 4), it means that the participant will make significant progress very fast but it will still take some time to become an expert. With low values (e.g., 1 or 1.5), the performance increases more slowly. An example of maturity curve is presented figure 2 . 


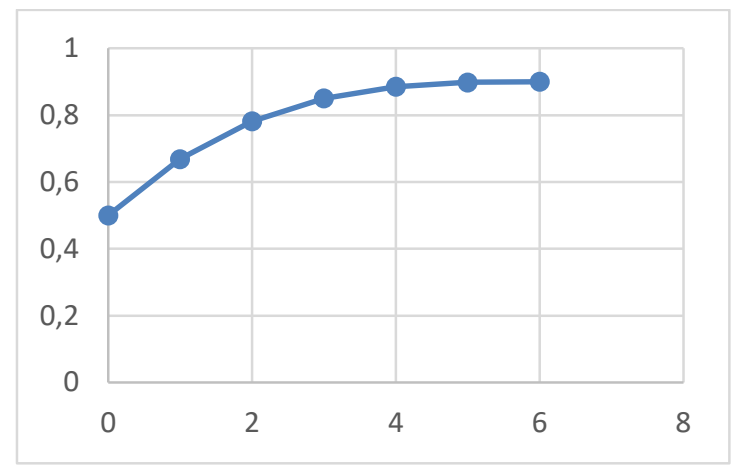

Figure 2: Example of maturity curve $\left(\mathrm{P}_{\max }(\mathrm{x})=0.9, \mathrm{P}_{\mathrm{i}}(\mathrm{x})=0.5, \mathrm{n}_{0}=6, \mathrm{k}=3\right)$.

\subsection{Integrating behavioral competencies}

Maturity models must be used at the beginning of the simulations. When sufficient data are available, probabilities can be determined using statistical tools. However, as explained before, statistics do not take training and individual differences into account. Maturity models can be used instead but the problem is to find a way to fix all parameters.

An important issue is to take individual capabilities into consideration. Some persons may indeed perform better than others for the first training session or their performance may increase more rapidly after several trials. An interesting approach is to consider behavioral competencies as they are defined by NASA and to adapt training sessions according to skills' requirements (Chator 2016). A global score can be calculated (not proposed in this study) in order to define different categories or profiles of participants. Then, statistics can be performed in each category to determine the exact values for $\mathrm{P}_{\max }(\mathrm{x})$, $\mathrm{P}_{\mathrm{i}}(\mathrm{x}), \mathrm{n}_{0}$ and $\mathrm{k}$. Interestingly, taking individual capabilities into consideration has also been proposed by Suhir with the Human Capacity Factor model (Suhir 2013). Both methods are indeed based on a similar probabilistic predictive modeling approach.

\section{Conclusion}

The proposed method is based on the use of Bayesian networks and Endsley's SA demons to predict situation awareness errors in training simulations. A key idea is to use a maturity model that allows simple calculations of probabilities even during the first simulation sessions. The drawback of this method is the parameters that have to be set empirically. However, these parameters can be linked to the cognitive and psychological profile of the participant. It is proposed here to use NASA behavioral competency model but other methods can be used.

This method is currently considered for several applications. The first one will be the Mars Explore simulations, in which two teams collaborate and a simulated vehicle is used to explore a region and collect Martian rocks. We hope to present the first results and an accurate assessment of the method in a near future.

\section{References}

Baoping C., Yonghong L., Yunwei Z., Qian F., Zengkai L. and Xiaojie T. (2013) 'A dynamic Bayesian networks modeling of human factors on offshore blowouts', Journal of Loss Prevention in the Process Industries, Vol. 26 No. 4, July 2013, pp. 639-649.

Barua S., Gao X., Pasman H. and Mannan S. (2016) 'Bayesian network based dynamic operational risk assessment', Journal of Loss Prevention in the Process Industries, Vol. 41, May 2016, pp. 399-410. 
Bedny, G.Z., Karwowski, W., Bedny, I. (2014), 'Applying Systemic-Structural Activity Theory to Design of Human-Computer Interaction Systems', CRC Press Taylor and Francis.

Chator O. and Salotti J.M. (2016), Skills and Human Engineering Issues at Task Design, Proceedings of the AHFE 2016 International Conference on Advances in Human Factors, Business Management, Training and Education, Walt Disney World®, Florida, USA, July 27-31.

De Winter, J. C., Happee, R., Martens, M. H., \& Stanton, N. A. (2014) 'Effects of adaptive cruise control and highly automated driving on workload and situation awareness: A review of the empirical evidence' Transportation research part F: traffic psychology and behaviour, 27, 196-217.

Endsley, M. R., \& Garland, D. J. (Eds.) (2000) 'Situation awareness analysis and measurement', Mahwah, NJ: Lawrence Erlbaum.

Endsley, M. R., \& Jones, D. G. (2016) 'Designing for situation awareness: An approach to user-centered design' ( $2^{\text {nd }}$ ed.), London: Taylor \& Francis.

Groth K. and Mosleh A. (2011) 'Development and Use of a Bayesian Netowrk to Estimate Human Error Probability, proceedings of the International Topical Meeting on Probabilistic Safety Assessment and Analysis Wilmington, NC, USA, March 13-17, 2011.

Kaber D. B., Riley J. M., Hyatt J. R., Reynolds J. P. (2006) 'Assessing infantry soldier situation awareness in virtual environment-based training of urban terrain operations', in Proceedings of the International Ergonomics Association Triennial Conference (pp. 1-6). Maastricht, The Netherlands: Elsevier.

Lemmer J. F. and Gossing D. E. (2005) 'Recursive Noisy OR-A Rule for Estimating Complex Probabilistic Interactions', IEEE Transactions on Cybernetics, Vol .34 No. 6, pp. 2252 - 2261, January 2005.

Mkrtchyan, L., Podofillini, L., \& Dang, V. N. (2015), 'Bayesian belief networks for human reliability analysis: A review of applications and gaps', Reliability engineering \& system safety, Vol. 139, pp. 116.

Muniz, E., Stout, R., Bowers, C., \& Salas, E. (1998), 'A methodology for measuring team situational awareness: situational awareness linked indicators adapted to novel tasks (SALIENT)' NATO human factors and medicine panel on collaborative crew performance in complex systems, Edinburgh, North Atlantic Treaties Organisation, Neuilly-sur-Seine, 20-24.

NASA (2008) 'International Space Station Human Behavior and Performance Competency Model', NASA/TM-2008-214775, Vol. 2, Langley, USA.

Park C.Y., Blackmond K., Paulo Costa L., Matsumoto S. (2013) Multi-entity Bayesian Networks Learning in Predictive Situation Awareness, proceedings of the $18^{\text {th }}$ International Command and Control Research and Technology Symposium C2 in Underdevloped, Degraded and Denied Operational Environments, June 2013, Alexandria, Virginia, USA.

Pearl J. (1988) 'Probabilistic Reasoning in Intelligent Systems: Networks of Plausible Inference', Morgan Kaufmann, 1988. 
Salotti J.M. (2010) 'Noisy-Or nodes for conditioning models', Lecture Notes in Artificial Intelligence, Vol. 6226, pp. 458-467, Springer, from "Simulation of Adaptive Behaviors", (SAB 2010), Paris, 24-28 August, 2010.

Salotti J.M. and Suhir E. (2014) 'Manned Missions to Mars: Minimizing Risks of Failure', Acta Astronautica, Vol. 93, pp. 148-161.

Sarter, N. B., \& Woods, D. D. (1991) 'Situation awareness: A critical but ill-defined phenomenon', The International Journal of Aviation Psychology, Vol. 1, No. 1, pp. 45-57.

Suhir, E. (2013), 'Miracle-on-the-Hudson: quantitative aftermath', International Journal of Human Factors Modelling and Simulation, Vol. 4, No. 1, pp. 35-62.

Suhir, S., Lini, S., Bey, C., Salotti, J.M., Hourlier S. and Claverie, B. (2015), 'Probabilistic modelling of the concept of anticipation in aviation', Theoretical Issues in Ergonomics Science, Vol. 1, No.1, pp. 69-85. 